\title{
Monitoring Alfvén Cascades with Interferometry on the JET Tokamak
}

\author{
S. E. Sharapov, ${ }^{1}$ B. Alper, ${ }^{1}$ J. Fessey, ${ }^{1}$ N. C. Hawkes, ${ }^{1}$ N. P. Young, ${ }^{1}$ R. Nazikian, ${ }^{2}$ G. J. Kramer, ${ }^{2}$ D. N. Borba, ${ }^{3}$ \\ S. Hacquin, ${ }^{3}$ E. De La Luna, ${ }^{4}$ S. D. Pinches,${ }^{5}$ J. Rapp, ${ }^{6}$ D. Testa,${ }^{7}$ and JET-EFDA Contributors* \\ ${ }^{1}$ Euratom/UKAEA Fusion Association, Culham Science Centre, Abingdon OX14 3DB, United Kingdom \\ ${ }^{2}$ Princeton Plasma Physics Laboratory, Princeton, New Jersey 08543-0451 USA \\ ${ }^{3}$ Association Euratom/IST, Centro de Fusão Nuclear, Av. Rovisco Pais, 1049-001 Lisboa, Portugal \\ ${ }^{4}$ Association Euratom-CIEMAT, Lab. Nacional de Fusion, A. Complutense 22, Madrid, Spain \\ ${ }^{5}$ Max-Plank Ins. Plasmaphys, Euratom Association, Boltzmannstraße 2, 85748 Garching, Germany \\ ${ }^{6}$ Forschungszentrum Jülich GmbH, Euratom Association, 52425 Jülich, Germany \\ ${ }^{7}$ CRPP/EPFL, Association Euratom-Confederation Suisse, 1015 Lausanne, Switzerland
}

(Received 8 March 2004; published 12 October 2004)

\begin{abstract}
A microwave interferometry technique is applied for the first time for detecting a discrete spectrum of Alfvén cascade (AC) eigenmodes excited with fast ions in reversed magnetic shear plasmas of the Joint European Torus. The interferometry measurements of plasma density perturbations associated with ACs show an unprecedented frequency and time resolution superior to that obtained with external magnetic coils. The measurements of ACs are used for monitoring the evolution of the safety factor and density of rational magnetic surfaces in the region of maximum plasma current.
\end{abstract}

DOI: 10.1103/PhysRevLett.93.165001

PACS numbers: 52.55.Fa, 42.87.Bg, 52.35.Bj, 52.55.Hc

Magnetohydrodynamic (MHD) spectroscopy that determines plasma characteristics via exciting and observing shear Alfvén eigenmodes (AEs) at low power was proposed at the very beginning of magnetic nuclear fusion research [1]. During the past decade, the development of techniques for exciting toroidal Alfvén eigenmodes (TAEs) [2,3] with external antennas and with super-Alfvénic ions has increased the quality of information that MHD spectroscopy can deliver via the measurements of these AEs [4-7]. More recently, a new class of frequency-sweeping discrete AEs was detected in JT-60U [8], Joint European Torus (JET) [7], and Tokamak Fusion Test Reactor (TFTR) [9] "advanced" tokamak plasma. These modes were called Alfvén cascades (ACs) $[7,10,11]$ and their applications for diagnosing the magnetic field topology have been assessed in [7,11-13]. The advanced scenarios in tokamaks operate at a reduced inductive current and are investigated to achieve longer plasma discharges and ultimately to reach steady-state tokamak operation at high fusion performance. These scenarios are focused on developing internal transport barriers (ITBs) that determine regions in plasmas where an improved insulation of the thermal plasma is achieved (see, e.g., [12,13]). ITBs are best triggered by applying auxiliary plasma heating before the inductive current has fully penetrated into the plasma, so that the topology of the equilibrium magnetic field $\mathbf{B}$ is optimized by varying the safety factor $q(r) \equiv \mathbf{B} \cdot \nabla \boldsymbol{s} / \mathbf{B} \cdot \nabla \theta$, which measures the "twist" of the magnetic field lines $\mathbf{B}$ as a function of the minor radius $r$ of the torus. Here, $r$ is constant on magnetic flux surfaces, and $(\theta, \varsigma)$ are appropriate poloidal and toroidal angle coordinates. It was experimentally found on JET $[12,13]$ that ITBs are triggered at a lower power of the auxiliary heating in plasmas with nonmo- notonic $q(r)$ profiles, i.e., with reversed magnetic shear, $S \cong(r / q)(d q / d r)<0$, in the plasma core. It was also established that the so-called "ITB triggering event," observed as a sudden increase in the slope of electron temperature $d T_{e} / d t$ (as well as $d T_{e} / d r$ ), usually starts at magnetic surface $r=r_{\min }$ corresponding to a minimum $q$ value $\left(q_{\min }\right)$ of the $q(r)$ profile at the time when $q_{\min }(t)$ passes an integer value [13]. A possible interpretation of the strong link between integer $q$ values and the spontaneous improvement of plasma confinement was given recently in terms of a reduced turbulent transport of the plasma due to the depleted density of rational magnetic flux surfaces around integer $q$ values $[14,15]$. Development of diagnostics, which can determine the time evolution of $q_{\min }(t)$, is paramount for both reliable and reproducible scenarios with ITBs and for investigating the transport properties of the layer surrounding $q_{\text {min }}$. MHD spectroscopy based on exciting the Alfvén cascades is one of the possible options for diagnosing the temporal evolution of $q_{\min }(t)$, since the AC eigenfrequencies $\omega_{A C}$ trace the evolution of $q_{\min }(t)[7,10,11]$ as

$$
\frac{d}{d t} \omega_{A C}(t) \approx m \frac{V_{A}}{R_{0}} \frac{d}{d t} q_{\min }^{-1}(t) .
$$

Here, $m$ is poloidal mode number of an $\mathrm{AC}, R_{0}$ is major radius of tokamak, $V_{A}=|\mathbf{B}| /\left(4 \pi n_{0} m_{i}\right)^{1 / 2}$ is the Alfvén velocity, $m_{i}$ is ion mass, and $n_{0}$ is the plasma density. In contrast to the motional Stark effect (MSE), which uses neutral beam injection (NBI) and provides measurement of entire $q(r)$ profile, the MHD spectroscopy based on (1) uses ion cyclotron resonance heating (ICRH) for exciting ACs and it provides accurate measurement of $q_{\min }(t)$. In the past, MHD spectroscopy was entirely reliant upon the $\mathrm{AC}$ measurements with external magnetic pick-up coils 
alone [7,11]. It was found recently [9] that reflectometry measurements of the density perturbations $\delta n$ associated with displacement $\xi$ of a shear Alfvén mode,

$$
\frac{\delta n}{n_{0}}=-\nabla \cdot \xi-\xi \cdot \frac{\nabla n_{0}}{n_{0}} \cong\left(\frac{-2 \hat{\mathrm{R}}}{\mathrm{R}_{0}^{2}}+\frac{\hat{\mathrm{n}}}{\mathrm{L}_{\mathrm{n}}}\right) \cdot \xi,
$$

can provide more information on core-localized ACs than the magnetic pick-up coils.

This Letter reports the first interferometry measurements of ACs driven with ions accelerated by ICRH in plasmas of the JET tokamak. This interferometry technique, which detects electron density perturbations associated with the ACs, shows an unprecedented frequency and time resolution of the eigenmodes, far superior to that obtained by external magnetic coils or by the electroncyclotron emission (ECE) radiometer reported earlier in $[7,11]$.

In order to compare both the external and internal measurements of ACs on JET, a multichannel O-mode reflectometer/interferometer, a multichannel electroncyclotron emission radiometer, and external magnetic pick-up coils were set up for measuring perturbed density, perturbed electron temperature, and perturbed magnetic field, respectively. The reflectometer/interferometer on JET is located at the outer side of the torus (at major radius $R \approx 4 \mathrm{~m}$ ) and it views the plasma horizontally through the magnetic axis with the line-of-sight perpendicular to the magnetic axis. Each channel of the system has two Gunn oscillators, the frequencies of which differ by $10.7 \mathrm{MHz}$, which is maintained by a phase-locked loop [16]. The microwave beam propagating through the plasma undergoes a change in amplitude and a shift in phase due to the variation of the refractive index $N(R)$ caused by the electron density $N(R)=[1-$ $\left.n_{e}(R) / n_{e}^{\text {crit }}\right]^{1 / 2}$. Here, the plasma electron density is a sum of equilibrium density and the perturbed density caused by AEs, $n_{e}(R)=n_{0}(R)+\delta n(R)$, and $n_{e}^{\text {crit }}$ is critical density determined for microwaves with frequency $\omega$ by the condition $\omega=\omega_{p e}$, where $\omega_{p e}$ is the plasma frequency. The signals associated with $\delta n$ are obtained from the amplitude $A$ and the change of the phase $\phi$ of microwave beams by comparing the beam propagating through the plasma with the reference beam outside the plasma. The beam propagating through the plasma is reflecting from the inner wall of the torus (at $R \approx 2 \mathrm{~m}$ ) in the interferometry regime and from the plasma in the reflectometry regime. The receiver measuring the returned beam is connected to a digital converter with a sampling rate $1 \mathrm{MHz}$ and the data for $A \cos \phi$ and $A \sin \phi$ are recorded with 14 bit effective resolution for $3 \mathrm{~s}$ during discharges.

Figure 1(a) shows time traces of the input power and the plasma parameters from a $B_{T} \approx 2.75 \mathrm{~T}, I_{p} \approx 1.7 \mathrm{MA}$ JET discharge in which lower hybrid current drive (LHCD) was applied during the current ramp-up phase
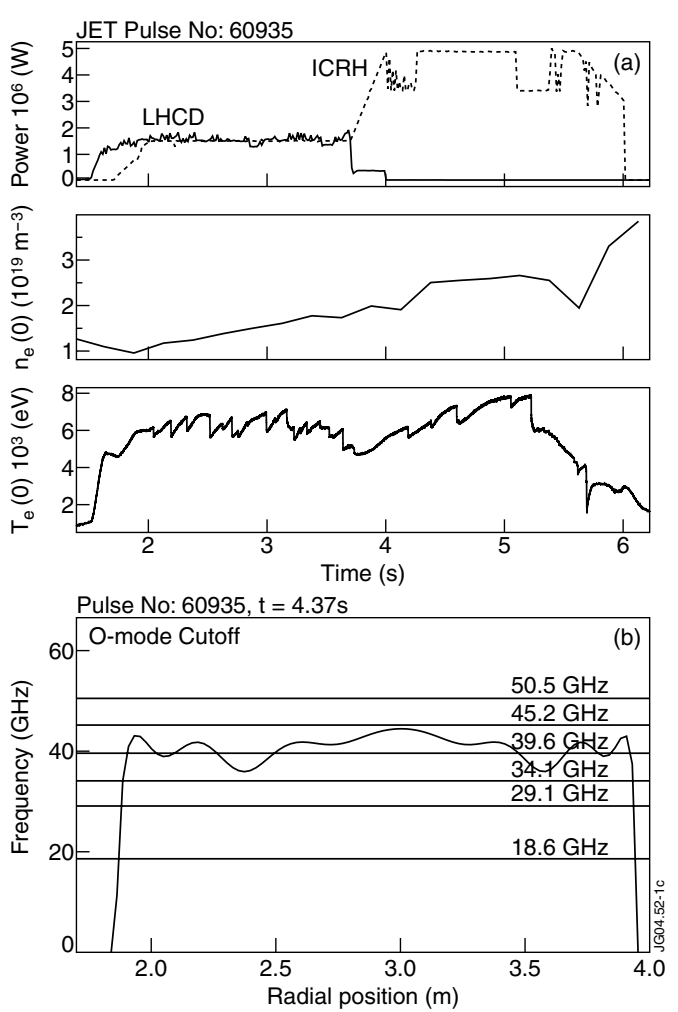

FIG. 1. (a) Time traces of LHCD and ICRH power, on-axis electron density, and central electron temperature in JET discharge No. 60935. (b) Frequency of the O-mode cutoff as a function of major radius calculated from the measured electron density profile together with frequencies of the six microwave beams launched from $R \approx 4 \mathrm{~m}$.

in order to obtain a reversed-shear magnetic configuration. ICRH power was used for accelerating hydrogen minority ions to energies high enough for the ions to resonate with shear Alf vén waves. Figure 1(b) shows the fixed frequencies of the six-channel microwave beams used in the experiment versus the cutoff frequency determined by the plasma density profile. These beams operated in the interferometry regime if the maximum plasma density was below $0.43,1.06,1.44,1.94,2.54$, or $3.16\left(\times 10^{19} \mathrm{~m}^{-3}\right)$, respectively, for each of the beams above. Figure 2 shows both the interferometry and the external magnetic coil measurements of Alfvén modes excited by the fast ions in this discharge. The Alfvén frequency spectrum consists of many frequencysweeping discrete modes observed in the frequency band from $40 \mathrm{kHz}$ to the toroidal Alfvén eigenmode frequency range, $140 \mathrm{kHz}$, in agreement with the wellestablished characteristics of the Alfvén cascades [10,11]. Figure 2 (top) shows the interferometry measurements of the perturbed density associated with the modes, indicating that very high resolution in both time and frequency with high sensitivity can be achieved so that ACs with toroidal mode numbers up to $n=16$ (inferred from the slope $\left.d \omega_{A C} / d t\right)$ are observed. In comparison, the mag- 


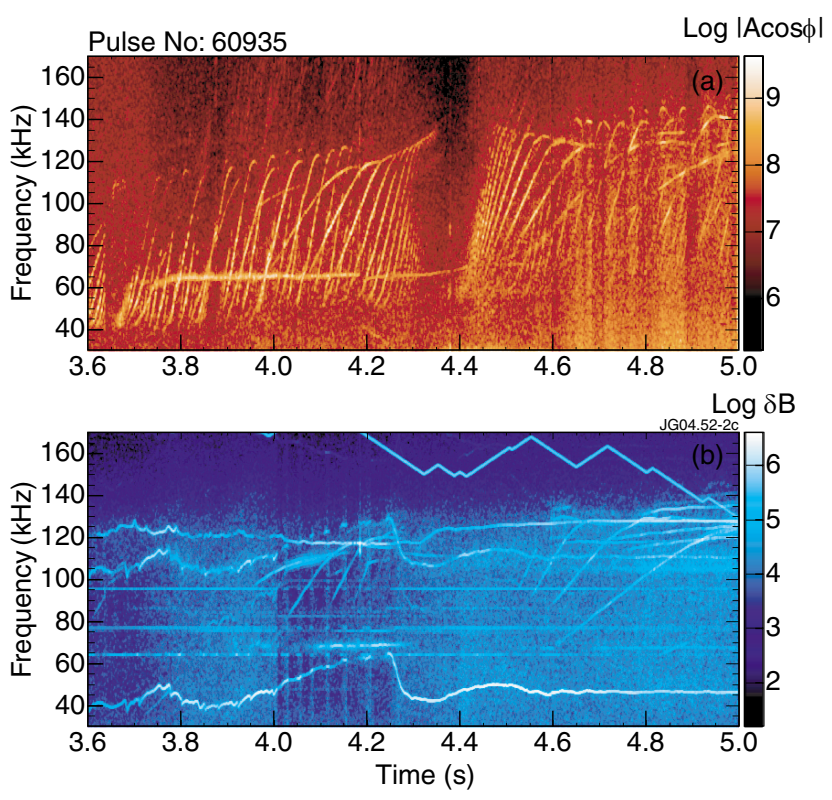

FIG. 2 (color). Fourier spectrograms showing Alfvén cascades with different toroidal and poloidal mode numbers in the discharge shown in Fig. 1. Top: interferometry measurements with microwave beam of $45.2 \mathrm{GHz}$. Bottom: measurements with external magnetic pick-up coil.

netic probe data shown in Fig. 2 (bottom) only detected ACs with mode numbers up to $n=5$ and during a much shorter time window. The physics interpretation of the data seen by the interferometry is independent of the choice between amplitude $A$ and the phase shift $\phi$ as they both show the ACs. However, the clearest images of the ACs were obtained on spectrograms showing amplitude $A$ and signals $A \cos \phi$ or $A \sin \phi$ due to the way the signals are recorded on JET. In accordance with [7,11], an Alfvén cascade starts to exist if the condition $m-$ $n q_{\text {min }}(t)=0$ becomes valid, where both $m, n$ are integers. It follows then, that for $q_{\min }(t)$ decreasing in time ACs of different mode number $m, n$ are excited one by one, scanning the condition $m / n=q_{\min }(t)$ in time. Figure 2 (top) is then showing the sequence of ACs associated with all possible rational values through which $q_{\min }(t)$ passes. Therefore, monitoring of the value of $q_{\mathrm{min}}(t)$ with a very high accuracy, up to $\Delta q_{\min } \approx 1 / 16$, can be performed based on the interferometry measurements. The appearance times of the ACs can be also used as a diagnostics of rational magnetic surfaces passing through a layer at $r \approx$ $r_{\min }$ associated with $q_{\min }(t)$. In particular, Fig. 2 (top) shows clearly that at $t \approx 4.4 \mathrm{sec}$, ACs of all possible mode numbers are excited simultaneously, indicating the appearance of $q_{\min }(t)=$ integer [7], and a clear gap in the density of the ACs observed just before this time shows the depletion of the rational $m / n$ values as discussed in $[14,15]$.

A series of JET discharges similar to that shown in Fig. 1 was performed with ICRH power scanned from its highest level of $5 \mathrm{MW}$ down to 1.4 MW. High quality data similar to that presented in Fig. 2 (top) was obtained from the interferometer even at the lowest level of ICRH power. On the other hand, the external magnetic coils were capable of detecting only a much smaller number of modes. Because of the lower level of ICRH power than in JET discharges described in [7,11], the multichannel ECE radiometer did not see any modes. In order to investigate the interferometry-to-reflectometry transition, the plasma density was gradually increased by applying $2 \mathrm{MW}$ of NBI to a JET discharge with a scenario similar to that in Fig. 1. Simultaneous measurements of the ACs and the $q(r)$ profile via MSE were performed. In the presence of the NBI, which fuels the plasma, the plasma density increased slowly from $2.5 \times 10^{19} \mathrm{~m}^{-3}$ to $3.3 \times 10^{19} \mathrm{~m}^{-3}$, so that two higher-frequency channels of the O-mode reflectometer came into reflection from layers at $R \approx 3.13 \mathrm{~m}$ and at $R \approx 3.42 \mathrm{~m}$ at a certain time when an AC was observed. On both channels, phase fluctuations were observed from the same AC with toroidal mode number $n=3$ and $m=6$ (determined from the set of magnetic coils). In order to estimate the density fluctuation level, the JET equilibrium was reconstructed with the MSE input for the weakly-reversed $q(r)$ profile. The $n=3$ AC mode was computed for this equilibrium with the ideal MHD MISHKA [17] and NOVA-K [18] codes, and the density fluctuation profile was used as input for a 1D reflectometry code [19]. The free parameter in this code, the density fluctuation level, $\delta n / n_{0}$, was adjusted to match the measured phase fluctuations. In this way, a density fluctuation level of $\delta n / n_{0} \approx 0.3 \%$ was obtained, from which value of magnetic fluctuations $\delta B / B_{0} \cong 2 \times$ $10^{-4}\left(f_{A C}[\mathrm{~Hz}] / 10^{5}\right)$ was inferred for the AC with frequency $f_{A C}$.

Further development of the interferometry technique followed this series of the dedicated experiments. By applying the interferometry technique routinely on JET, it was noted that the ACs are best observed in the interferometry if the microwave beam frequency is just above (by 10\%-20\%) the critical frequency determined by the maximum density of the plasma. This effect is explained by sensitivity of the response of the refraction index to the density $\delta n$ which takes the form $\delta N \cong-(\delta n / 2) \times$ $\left[n_{e}^{\text {crit }}\left(n_{e}^{\text {crit }}-n_{0}\right)\right]^{-1 / 2}$ when $\left(n_{e}^{\text {crit }}-n_{0}\right) / n_{e}^{\text {crit }} \ll 1$, i.e., the response is $\left[n_{e}^{\text {crit }} /\left(n_{e}^{\text {crit }}-n_{0}\right)\right]^{1 / 2}$ times stronger in this case than for $n_{0} \ll n_{e}^{\text {crit }}$. In discharges with a growing density, a multichannel approach used on JET was found to be very useful as the AC image is always seen on at least one interferometry channel. In such a way, the interferometry diagnostics of ACs allow a permanent monitoring of the ACs that provides reliable data for identifying exact timing of $q_{\min }(t)=$ integer events, which are most favorable for the ITB triggering. In order to expand the technique to higher density JET discharges, a higher-frequency microwave beam (at $63.80 \mathrm{GHz}$ ) was 

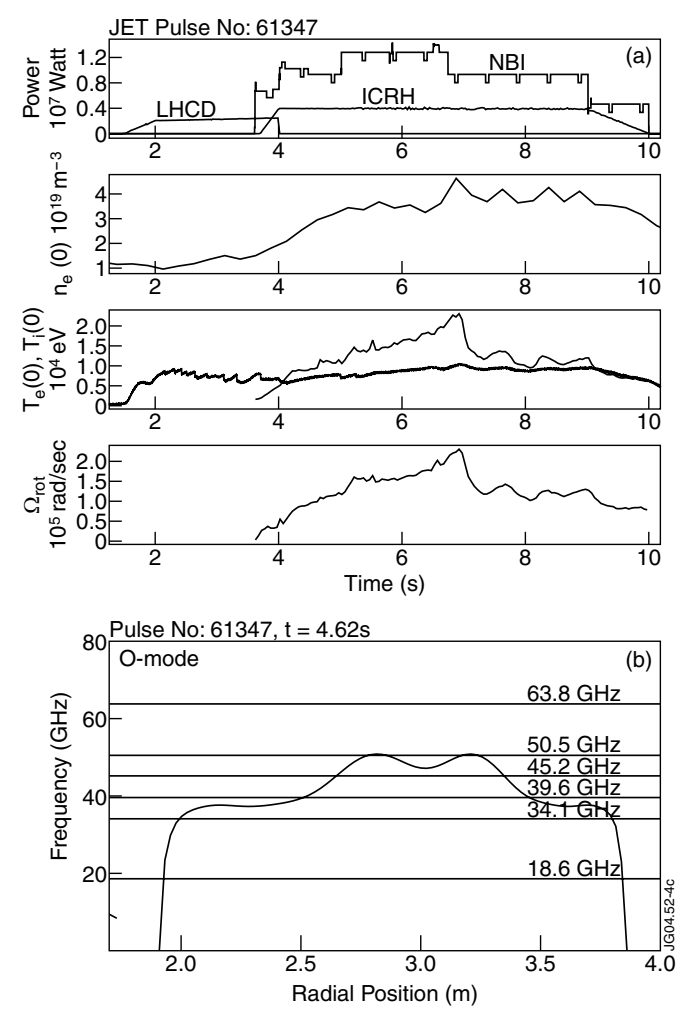

FIG. 3. (a) Time traces of LHCD, ICRH, and NBI power, onaxis plasma density, central electron and ion temperature, and toroidal plasma rotation in JET discharge No. 61347. (b) Frequency of the O-mode cutoff as a function of major radius together with frequencies of the six microwave beams.

added to the data acquisition therefore covering the range of the AC measurements to JET scenarios with high NBI power. Figure 3(a) shows time traces for a typical shearreversed JET discharge with strong NBI heating while Fig. 3(b) shows the microwave frequencies used in the experiments versus the cutoff profile. In this discharge, not only is the plasma density higher than in the discharge shown in Fig. 1, but also the plasma rotates toroidally at high speed, driven by NBI. The interferometry measurements obtained in this case are shown in Fig. 4. This data significantly differs from that shown in Fig. 2(a) as the modes now cover a much broader frequency band, up to $300 \mathrm{kHz}$. The broader frequency range is caused by the Doppler shift $\cong n \Omega_{\text {rot }}$ due to strong toroidal plasma rotation, up to $\Omega_{\text {rot }} \cong 2 \times 10^{5} \mathrm{~s}^{-1}$, and the high mode numbers of the ACs, up to $n \approx 10$.

In summary, a new interferometry technique was developed for monitoring the discrete spectrum of Alfvén cascade eigenmodes driven by ICRH-accelerated ions in advanced scenario of the JET tokamak. The interferometry measurements show an unprecedented frequency and time resolution of the eigenmodes and are used successfully for monitoring the evolution of the safety factor for developing scenarios with internal transport barriers. Similar techniques based on higher-frequency micro-

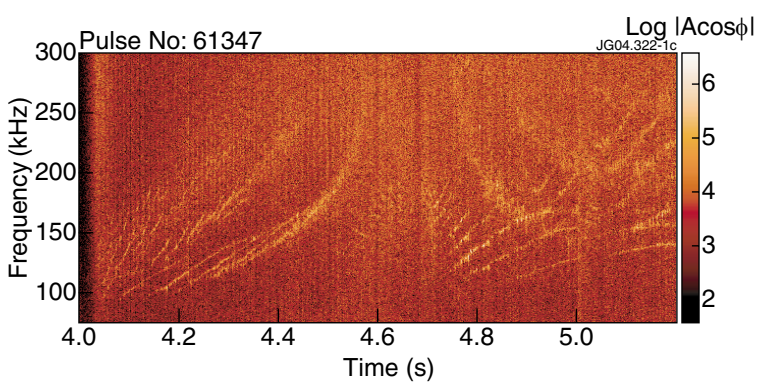

FIG. 4 (color). Interferometry measurement of Alfvén cascades with microwave beam of $63.8 \mathrm{GHz}$ in JET discharge No. 61347 shown in Fig. 3.

waves may be applied for diagnosing plasma of tokamak-reactors such as International Thermonuclear Experimental Reactor (ITER).

This work was partly funded by Euratom and by the UK Engineering and Physical Sciences Research Council. This work was performed under the European Fusion Development Agreement.

*Annex of J. Paméla et al., Fusion Energy 2002, Proceedings of the 19th IAEA Conference on Fusion Energy, Lyon, 2002.

[1] T. Stix, Phys. Fluids 1, 308 (1958).

[2] C.Z. Cheng, L. Chen, and M. S. Chance, Ann. Phys. (N.Y.) 161, 21 (1985).

[3] K.-L. Wong, Plasma Phys. Controlled Fusion 41, R1 (1999).

[4] J. P. Goedbloed et al., Plasma Phys. Controlled Fusion 35, B277 (1993).

[5] G. Kramer, C. Z. Cheng, Y. Kusama et al., Nucl. Fusion 41, 1135 (2001).

[6] A. Fasoli et al., Plasma Phys. Controlled Fusion 44, B159 (2002).

[7] S. E. Sharapov et al., Phys. Lett. A 289, 127 (2001).

[8] H. Kimura, Y. Kusama, M. Saigusa, et al., Nucl. Fusion 38, 1303 (1998).

[9] R. Nazikian et al., Phys. Rev. Lett. 91, 125003 (2003).

[10] H. L. Berk, D. N. Borba, B. N. Breizman et al., Phys. Rev. Lett. 87, 185002 (2001).

[11] S. E. Sharapov, B. Alper, H. L. Berk et al., Phys. Plasmas 9, 2027 (2002).

[12] E. Joffrin et al., Plasma Phys. Controlled Fusion 44, 1739 (2002).

[13] E. Joffrin et al., Nucl. Fusion 43, 1167 (2003).

[14] Y. Kishimoto et al., Plasma Phys. Controlled Fusion 41, A663 (1999).

[15] X. Garbet et al., Nucl. Fusion 43, 975 (2003).

[16] A. C. C. Sips and G. J. Kramer, Plasma Phys. Controlled Fusion 35, 743 (1993).

[17] A. B. Mikhailovskii et al., Plasma Phys. Rep. 23, 844 (1997).

[18] C. Z. Cheng, Phys. Rep. 211, 1 (1992).

[19] G. J. Kramer R. Nazikian, and E. Valeo, Plasma Phys. Controlled Fusion 44, L11 (2002). 